\title{
Avaliação da Pressão Venosa Periférica na Esclerose Sistêmica(*)
}

\section{Peripheral Venous Pressure in Systemic Sclerosis}

\author{
João Potério-Filho(1), Percival D. Sampaio-Barros ${ }^{(2)}$, Glória Maria Braga Potério ${ }^{(3)}$, \\ Adil M. Samara ${ }^{(4)}$ e João Francisco Marques Neto(4)
}

\section{RESUMO}

Objetivo: Estabelecer a utilidade da medida indireta da pressão venosa periférica (PVP) na avaliação de pacientes com esclerose sistêmica (ES), comparando com uma população controle normal. Métodos: Estudo prospectivo avaliando 18 pacientes esclerodérmicas do sexo feminino (sendo oito delas com úlcera cutânea isquêmica) submetidas à medida indireta da PVP (por pletismografia). Os dados foram comparados a um grupo controle normal de 18 mulheres hígidas, pareadas para idade. Resultados: Os níveis de pressão arterial foram semelhantes nos dois grupos. Os valores de PVP estavam significativamente diminuídos nos pacientes com ES $(58.9 \pm 11.6 \mathrm{mmHg}$ no grupo ES e $96.9 \pm 7.1 \mathrm{mmHg}$ no grupo controle; $\mathrm{p}<0.0001)$; no grupo com ES, os pacientes com úlceras cutâneas isquêmicas apresentaram níveis de PVP significativamente diminuídos comparando-se com os pacientes com ES sem úlceras (50.6 $\pm 10.8 \mathrm{mmHg}$ no grupo com úlceras e $65.5 \pm 7.2 \mathrm{mmHg}$ no grupo sem úlceras; $p=0.006$ ). Conclusões: Pode-se inferir que a diminuição nos níveis de PVP na ES parece estar associada a uma diminuição do fluxo sangüíneo, predispondo ao aparecimento de úlceras isquêmicas nestes pacientes.

Palavras-chave: Escleroderma sistêmica, pressão venosa periférica, úlcera cutânea.

\section{INTRODUÇÃO}

A disfunção vascular é um elemento-chave na esclerose sistêmica $(E S)^{(1,2)}$, e um sistema vascular ativado está freqüentemente associado a uma significativa morbidade na evolução da doença ${ }^{(3)}$. O fenômeno de Raynaud (FRy) com microulcerações de polpas digitais se constitui na

\section{ABSTRACT}

Objective: The aim of this prospective study was to establish the usefulness of indirect measurement of peripheral venous pressure (PVP) in the evaluation of patients with systemic sclerosis (SSC), comparing them with a normal control population. Methods: Eighteen female SSc patients (eight patients presenting cutaneous ischemic ulcers) were submitted to indirect measurement of the PVP (by plethysmography), and data were compared with a control group of 18 healthy women, paired by age. Results: Arterial pressure levels were similar in both groups. PVP levels were significantly decreased in the SSC patients $(58.9 \pm 11.6 \mathrm{mmHg}$ in the SSc group and $96.9 \pm 7.1$ $\mathrm{mmHg}$ in the control group; $p<0.0001)$; in the SSc group, patients with ischemic ulcers presented PVP levels significantly decreased compared with those patients without ulcers (50.6 $\pm 10.8 \mathrm{mmHg}$ in the ulcer group and $65.5 \pm 7.2 \mathrm{mmHg}$ in the group without ulcers; $p=0.006$ ). Conclusions: It can be concluded that decreased PVP in SSC is associated with a decrease in the blood flow of these patients, predisposing them to cutaneous ischemic ulcers.

Keywords: Systemic sclerosis, peripheral venous pressure, cutaneous ulcer.

manifestação vascular mais freqüente nos pacientes esclerodérmicos $^{(4,5)}$. Tradicionalmente, o envolvimento vascular na ES tem sido considerado como predominantemente microvascular, associado a um desbalanço entre a vasoconstrição e a vasodilatação, em favor da vasoconstrição ${ }^{(2,5)}$. Nos últimos anos, a presença de acometimento macrovascular tem sido observada de maneira crescente na $\mathrm{ES}^{(6-9)}$.

\footnotetext{
* Trabalho realizado na Disciplina de Cirurgia Vascular do Departamento de Cirurgia e na Disciplina de Reumatologia do Departamento de Clínica Médica da Faculdade de Ciências Médicas da Universidade Estadual de Campinas (FCM-UNICAMP). Recebido em 29/09/03. Aprovado, após revisão, em 14/01/04.

1. Professor Adjunto da Disciplina de Cirurgia Vascular. (FCM-UNICAMP).

2. Assistente-Doutor da Disciplina de Reumatologia. (FCM-UNICAMP).

3. Professora Livre-Docente do Departamento de Anestesiologia. (FCM-UNICAMP).

4. Professor Titular da Disciplina de Reumatologia. (FCM-UNICAMP).
}

Endereço para correspondência: Dr. Percival D. Sampaio-Barros. Disciplina de Reumatologia, Departamento de Clínica Médica. Faculdade de Ciências Médicas Universidade Estadual de Campinas (FCM-UNICAMP). Barão Geraldo, Campinas, SP, CEP 13081-970. E-mail: psbarros@fcm.unicamp.br 
O estabelecimento de técnicas de medida acurada e reprodutível da função vascular são elementos muito importantes na compreensão da complexa fisiopatologia da doença.

Os autores investigaram a importância da medida indireta da pressão venosa periférica (PVP) em pacientes esclerodérmicos, comparado a um grupo controle saudável.

\section{MATERIAL E MÉTODOS}

Os autores realizaram um estudo prospectivo analisando a pressão arterial (PA) e a PVP num grupo de 18 pacientes com diagnóstico de ES de acordo com os critérios preliminares do Colégio Americano de Reumatologia ${ }^{(10)}$, subdivididos em formas clínicas difusa (ESd) e limitada (ESl) de acordo com os critérios de LeRoy e colaboradores ${ }^{(11)}$. Todos os pacientes eram do sexo feminino, com idade variando entre 16 e 46 anos (com média de idade 34 anos). Oito pacientes apresentavam úlceras cutâneas isquêmicas no momento da medida das pressões. Os dados foram comparados com as mesmas medidas obtidas num grupo controle saudável constituído por 18 mulheres, pareadas por idade.

As pressões foram avaliadas numa temperatura variando entre $20^{\circ} \mathrm{C}$ e $25^{\circ} \mathrm{C}$. A PA foi medida no membro superior esquerdo com o paciente em posição supina utilizando-se um ultra-som com doppler.

A PVP foi avaliada com o paciente na posição supina, através da pletismografia de oclusão venosa. A oclusão com um cuff de esfigmomanômetro (cuff número 1) foi aplicada no braço esquerdo, enquanto um cuff de leitura (cuff número 2) foi aplicado no antebraço distal esquerdo. Num primeiro passo, a PA era obtida utilizando-se o cuff número 1 e o ultra-som com doppler sobre a artéria radial no nível do punho. Num segundo passo, pressões eram aplicadas em ambos os cuffs ao mesmo tempo, mantendo-se a pressão no cuff $110 \mathrm{mmHg}$ maior que a pressão no cuff 2 . Desta forma, a pressão ia crescendo lentamente até que a linha de registro do pletismógrafo mostrasse um padrão estável; esta pressão obtida foi considerada como a PVP. Utilizando-se a técnica pletismográfica, a linha de registro cairia se a PVP fosse ultrapassada.

A conferência invasiva da PVP foi realizada em cinco voluntárias saudáveis do sexo feminino após a leitura com a técnica pletismográfica, utilizando-se uma agulha (Abbocate 16) na veia ulnar conectada diretamente ao manômetro. Nenhuma diferença estatística foi observada entre o método pletismográfico e a medida direta da PVP.

\section{RESULTADOS}

Os níveis da PA sistólica foram similares nos dois grupos $(121.1 \pm 9.6 \mathrm{mmHg}$ no grupo ES e $116.1 \pm 11.6 \mathrm{mmHg}$ no grupo controle; $\mathrm{p}=0.168$ ). Hipertensão arterial não foi referida por nenhuma paciente.

Os valores da PVP estavam significativamente diminuídos nas pacientes com ES $(58.9 \pm 11.6 \mathrm{mmHg}$ no grupo ES e $96.9 \pm 7.1 \mathrm{mmHg}$ no grupo controle; $\mathrm{p}<0.0001)$. As pacientes esclerodérmicas com úlceras isquêmicas apresentaram níveis de PVP significativamente diminuídos comparando-se com as pacientes que não apresentavam úlceras isquêmicas no momento da aferição da PVP $(50.6 \pm 10.8$ $\mathrm{mmHg}$ no grupo com úlceras e $65.5 \pm 7.2 \mathrm{mmHg}$ no grupo sem úlceras; $\mathrm{p}=0.006$ ).

Com respeito ao índice PVP/PA, estava significativamente diminuído no grupo ES $(0.480 \pm 0.092$ no grupo ES e 0.840 \pm 0.042 no grupo controle; $p<0.0001)$. No grupo ES, o índice PVP/PA estava significativamente diminuído no grupo com úlceras isquêmicas $(0.414 \pm 0.075)$ comparado ao grupo sem úlceras $(0.538 \pm 0.058)(\mathrm{p}=0.003)$.

Os valores das pressões em todo o grupo esclerodérmico comparados àqueles do grupo controle estão especificados na tabela 1, enquanto que os valores das pressões nos dois grupos com ES (com e sem úlceras cutâneas isquêmicas) estão demonstrados na tabela 2 .

TABELA 1

NÍVEIS PRESSÓRICOS MÉDIOS: COMPARAÇÃO ENTRE PACIENTES COM ES E CONTROLES

\begin{tabular}{lccc}
\hline Pressões & ES & Controles & $\mathrm{p}$ \\
\hline PA $(\mathrm{mmHg})$ & $121.1 \pm 9.6$ & $116.1 \pm 11.6$ & NS \\
PVP $(\mathrm{mmHg})$ & $58.9 \pm 11.6$ & $96.9 \pm 7.1$ & $<0.0001$ \\
PVP / PA & $0.480 \pm 0.092$ & $0.840 \pm 0.042$ & $<0.0001$ \\
\hline
\end{tabular}

$\mathrm{ES}=$ esclerose sistêmica; $\mathrm{PA}=$ pressão arterial; $\mathrm{PVP}=$ pressão venosa periférica; NS = não significativo; valores expressos como média e desvio padrão; teste T pareado $\mathrm{p}<0.05$ ou similar

TABELA 2

NÍVEIS PRESSÓRICOS MÉDIOS: COMPARAÇÃO ENTRE PACIENTES COM ES COM E SEM ÚLCERAS ISQUÊMICAS

\begin{tabular}{lccc}
\hline Pressões & ES com úlceras & ES sem úlceras & $p$ \\
\hline PA (mmHg) & $120.0 \pm 10.7$ & $122.0 \pm 9.2$ & NS \\
PVP $(\mathrm{mmHg})$ & $50.6 \pm 10.8$ & $65.5 \pm 7.2$ & 0.006 \\
PVP / PA & $0.414 \pm 0.075$ & $0.538 \pm 0.058$ & 0.003 \\
\hline
\end{tabular}

$E S=$ esclerose sistêmica; $P A=$ pressão arterial; $P V P=$ pressão venosa periférica; $\mathrm{NS}=$ não significativo; valores expressos como média e desvio padrão; teste T pareado $\mathrm{p}<0.05$ ou similar 


\section{DISCUSSÃO}

Embora o FRy primário não curse com úlceras isquêmicas digitais, os pacientes com FRy e ES podem progredir para isquemia digital irreversível com microcicatrizes, ulcerações, e até mesmo gangrena. Em sua recente proposição de critérios de classificação para a ES precoce, LeRoy e Medsger ${ }^{(12)}$ enfatizaram a importância do FRy, documentado objetivamente como o principal critério clínico para o diagnóstico das formas iniciais da ES limitada. Os eventos iniciais que geram o complexo processo da disfunção vascular e da ativação endotelial na ES ainda não estão completamente elucidados. Muitas substâncias estão envolvidas na regulação do tônus vascular ${ }^{(1-5 ; 13)}$, sempre com um franco predomínio do tônus vasoconstritor.

Estudos recentes examinaram as respostas do fluxo sangüíneo digital, medido pela via oclusiva pletismográfica, em 10 pacientes com FRy e ES e 10 controles saudáveis, e mostraram respostas diminuídas à metacolina (uma droga vasodilatadora dependente do endotélio) e à clonidina (um agonista $\alpha_{2}$-adrenérgico) nos pacientes com ES, bem como respostas similares ao nitroprussiato de sódio (uma droga vasodilatadora independente do endotélio) ${ }^{(14)}$.

A medida da pressão capilar em seres humanos utilizandose um método oclusivo venoso ${ }^{(15)}$ representa um método fácil e prático de estimar a PVP, especialmente pelo fato de ser um método não invasivo. Neste estudo, os autores

\section{REFERÊNCIAS}

1. LeRoy EC: Systemic sclerosis: A vascular perspective. Rheum Dis Clin North Am 22: 675-94, 1996.

2. Herrick AL: Vascular function in systemic sclerosis. Curr Opin Rheumatol 12: 527-33, 2000.

3. Kahaleh MB, LeRoy EC: Autoimmunity and vascular involvement in systemic sclerosis (SSc). Autoimmunity 31: 195-214, 1999.

4. Wigley FM, Flavahan NA: Raynaud's phenomenon. Rheum Dis Clin North Am 22: 765-81, 1996.

5. Guiducci S, Pignone A, Matucci-Cerinic M: Raynaud's phenomenon in systemic sclerosis. Em Clements PJ, Furst DE (Editores): Systemic sclerosis. $2^{a}$ edição, Lippincott Williams \& Wilkins, Filadélfia, págs. 221-40, 2004.

6. Stafford L, Englert H, Gover J, Bertouch J: Distribution of macrovascular disease in scleroderma. Ann Rheum Dis 57: 476-9, 1998.

7. Ho M, Veale D, Eastmond C, Nuki, Belch J: Macrovascular disease in systemic sclerosis. Ann Rheum Dis 59: 39-43, 2000.

8. Taylor MH, McFadden JA, Bolster MB, Silver RM: Ulnar artery involvement in systemic sclerosis (scleroderma). J Rheumatol 29: 102-6, 2002.

9. Matucci-Cerinic M, Fiori G, Grenbaum E, Shoenfeld Y: Peripheral macrovascular involvement in systemic sclerosis. Em Clements PJ, observaram que os níveis de PVP estão significativamente diminuídos nos pacientes com ES, comparando-se com um grupo controle de mulheres saudáveis pareadas para a idade. A inclusão de pacientes esclerodérmicas com idade entre 16 e 45 anos visava excluir pacientes idosas e/ou com problemas vasculares prévios, relacionados ou não à ES. E foi ainda observado que os níveis da PVP eram ainda menores nos pacientes com ES que apresentavam úlcera cutânea isquêmica no momento da aferição das pressões. A causa primária desta diminuição da PVP nos pacientes com ES (especialmente naqueles com úlceras isquêmicas) ainda não é completamente compreendida, sendo necessários futuros estudos analisando os parâmetros bioquímicos e mecânicos que possam contribuir para uma diminuição dessa resposta aos vasodilatadores. Os autores demonstraram previamente que a compressão rítmica externa dos membros em pacientes com ES e úlceras cutâneas isquêmicas pode contribuir para a cicatrização das úlceras ${ }^{(16)}$. Além da importância do tratamento destas úlceras isquêmicas dolorosas na ES, também é importante selecionar os pacientes que apresentam risco maior de desenvolver estas úlceras. A medida indireta da PVP, obtida por métodos não invasivos, parece se constituir num instrumento útil na procura dos fatores predisponentes às úlceras isquêmicas na ES; novos estudos, com maior número de pacientes e seguimento de longo prazo, serão necessários, mas os resultados iniciais são animadores.

Furst DE (Editores): Systemic sclerosis. $2^{\mathrm{a}}$ edição, Lippincott Williams \& Wilkins, Filadélfia, págs. 241-7, 2004.

10. Masi AT, Rodnan GP, Medsger TA Jr, et al: Preliminary criteria for the classification of systemic sclerosis (scleroderma). Arthritis Rheum 23: 581-90, 1980.

11. LeRoy EC, Black C, Fleischmajer R, et al: Scleroderma (systemic sclerosis): classification, subsets and pathogenesis. J Rheumatol 15 : 202-5, 1988.

12. LeRoy EC, Medsger TA Jr: Criteria for the classification of early systemic sclerosis. J Rheumatol 28: 1573-6, 2001.

13. Kahaleh B, Matucci-Cerinic M: Raynaud's phenomenon and scleroderma: dysregulated neuroendothelial control of vascular tone. Arthritis Rheum 38: 1-4, 1995.

14. Freedman RR, Girgis R, Mayes MD: Endothelial and adrenergic dysfunction in Raynaud's phenomenon and scleroderma. J Rheumatol 26: 2386-8, 1999

15. Perry MA, Colebatch JG, Glover WE, Roddie IC: Measurement of capillary pressure in humans using a venous occlusion method. J Appl Physiol 60: 2114-7, 1986.

16. Potério Filho J, Sampaio-Barros PD, Parente JBHF, et al: Rhythmic external compression of the limbs: A method for healing cutaneous ulcers in systemic sclerosis. J Rheumatol 25: 1540-3, 1998. 